\title{
Recovery from COVID-19 Pneumonia in a Patient with Acute Severe Colitis
}

\author{
Karoline Soares Garcia ${ }^{a}$ Bruna Damásio Moutinho ${ }^{a}$ \\ Matheus Freitas Cardoso de Azevedo ${ }^{a} \quad$ Natalia Sousa Freitas Queiroz ${ }^{b}$

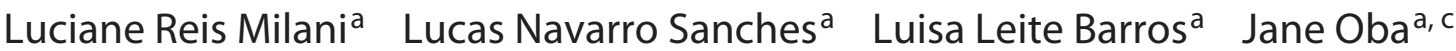 \\ Alexandre de Sousa Carlos ${ }^{a}$ Aderson Omar Mourão Cintra Damião ${ }^{a}$ \\ Aytan Miranda Sipahi ${ }^{a}$ d \\ ${ }^{a}$ Division of Gastroenterology and Hepatology, Department of Gastroenterology, Hospital das Clínicas, School \\ of Medicine, University of São Paulo, São Paulo, Brazil; b Department of Gastroenterology, Hospital das Clínicas, \\ School of Medicine, University of São Paulo, São Paulo, Brazil; ' Instituto da Criança e do Adolescente, Hospital das

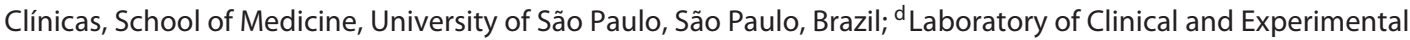 \\ Gastroenterology (LIM-07), Hospital das Clínicas, School of Medicine, University of São Paulo, São Paulo, Brazil
}

\section{Keywords}

Colitis · Coronavirus infections · COVID-19 · Inflammatory bowel disease $\cdot$ Ulcerative colitis

\footnotetext{
Abstract

Introduction: The coronavirus disease 2019 (COVID-19) pandemic has increased concern regarding SARS-CoV-2 infection in inflammatory bowel disease (IBD) patients, especially those on immunosuppressive therapies or with active disease. There are limited reports describing the clinical features of COVID-19 in an IBD population, and the impact of immunosuppression on the severity of the infection remains unclear. Case Report: A 33-year-old female patient with a long history of ulcerative colitis, poorly controlled, was admitted with COVID-19 a few days after being discharged from the hospital for treatment of acute severe ulcerative colitis. High-risk factors for COVID-19 complications, i.e., high-dose steroids (40 mg prednisone) and severe active disease, were present at admission. Despite the development
}

\begin{tabular}{ll}
\hline KARGER & $\begin{array}{l}\text { (c) } 2020 \text { The Author(s) } \\
\text { Published by S. Karger AG, Basel }\end{array}$ \\
karger@karger.com & This article is licensed under the Creative Commons Attribution- \\
Noncommercial-NoDerivatives 4.0 International License (CC BY- \\
NC-ND) (http://www.karger.com/Services/OpenAccessLicense). \\
Nsage and distribution for commercial purposes as well as any dis- \\
tribution of modified material requires written permission.
\end{tabular}

of extensive pulmonary involvement, the patient had a favorable outcome. Discussion: Management of IBD patients during the COVID-19 pandemic has been challenging. Measures to minimize the potential risk of SARS-CoV-2 infection, including strict social distancing and self-isolation, in the IBD population have been recommended, especially for highrisk patients. Although steroid tapering and persistence of biologics are advised by professional groups, the best treatment strategy for IBD patients presenting a flare during the outbreak has yet to be defined. @ 2020 The Author(s)

Published by S. Karger AG, Basel

\section{Introduction}

The coronavirus disease 2019 (COVID-19) pandemic is spreading quickly from China to the rest of the world and has led to $1,439,516$ confirmed cases and 85,711 deaths worldwide as of April 9, 2020 [1]. Specific populations, such as elderly, hypertensive, diabetic, and obese 
patients, as well as patients with malignancies, are at a greater risk for worse outcomes [2].

For patients with inflammatory bowel disease (IBD), especially those on immunosuppressive therapies or with active disease, the current pandemic requires several new considerations. Previous studies have shown that disease activity and exposure to thiopurines increase the risk of viral infections $[3,4]$. However, there is expert agreement that IBD patients are not at a greater risk of developing SARS-CoV-2 infection than the general population [5]. There are limited data regarding the clinical features of COVID-19 in an IBD population, and the impact of immunosuppression on the severity of COVID-19 remains uncertain. Thus, protective measures such as sheltering in place and strict social distancing are highly recommended for this special population.

\section{Case Report}

A 33-year-old female diagnosed with ulcerative pancolitis and primary sclerosing cholangitis in 2012 was admitted to the hospital on March 16, 2020, due to abdominal pain, bloody diarrhea (10 bowel movements per day), hyporexia, and vomiting that had started 2 weeks before. At an outpatient clinic, she was given sulfasalazine $4 \mathrm{~g} /$ day, topical mesalamine $1 \mathrm{~g} / \mathrm{day}$, and ursodeoxycholic acid $900 \mathrm{mg} /$ day. She was previously exposed to azathioprine, which was discontinued due to hepatotoxicity. The IBD team decided to initiate biological therapy. While she was awaiting laboratory tests, she presented to the emergency department with tachycardia (115 bpm), pale mucous membranes, abdominal distention, and diffuse tenderness. Initial laboratory tests revealed severe anemia (hemoglobin $6.5 \mathrm{~g} / \mathrm{dL}$ ), normal white blood cell and platelet counts, and an elevated C-reactive protein $(21.5 \mathrm{mg} / \mathrm{L})$. Albumin levels were reduced $(2.5 \mathrm{~g} / \mathrm{dL})$, and liver enzymes were within the baseline values for the patient, with no change in liver function tests. An abdominal computed tomography (CT) scan showed diffuse parietal bowel wall thickening, moderate loop distension, diffuse densification of the fat surrounding the cecum and ascending colon, a diffuse increase in the number of locoregional lymph nodes, and engorgement of the mesocolon and mesorectal vessels (Fig. 1). Fecal tests excluded Clostridioides difficile and other enteric pathogen infections, while biopsies obtained by flexible sigmoidoscopy excluded cytomegalovirus infection (Fig. 2). Therefore, the patient was diagnosed with acute severe colitis. She received a transfusion of red blood cells in the emergency department. Appropriate clinical treatment was initiated with corticosteroids (hydrocortisone at $300 \mathrm{mg} /$ day) and prophylactic anticoagulation (enoxaparin). Screening tests for biological therapy revealed a strongly positive tuberculin test $(15 \mathrm{~mm})$ and a normal chest CT scan (Fig. 3a), the setting for a latent tuberculosis. The patient had an excellent response to corticosteroids, with progressive clinical improvement, and was discharged on March 25th using prednisone at $40 \mathrm{mg} /$ day. The outpatient therapeutic plan was to start biological therapy after 30 days of treatment with isoniazid (9-month treatment schedule, according to Brazilian guidelines)

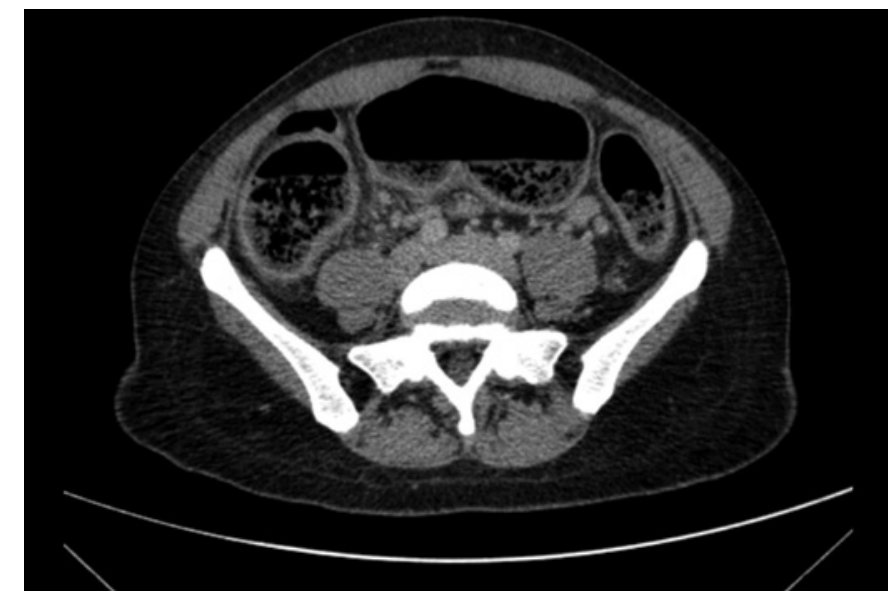

Fig. 1. Abdominal pelvic CT scan showing thickness of the colonic wall, moderate bowel distension, diffuse densification of the fat surrounding the cecum, and a diffuse increase in the number of locoregional lymph nodes.

[6]. One day after discharge, the patient started with symptoms of dry cough and runny nose. The symptoms progressively intensified and she was readmitted to the hospital 4 days later. At that point, the patient had mild dyspnea, but without oxygen desaturation. Considering the pandemic scenario, nasopharyngeal and oropharyngeal swabs revealed that she was SARS-CoV-2 PCR positive.

Additional laboratory tests documented: $\mathrm{Hb}, 9 \mathrm{~g} / \mathrm{dL}$; white blood cell count, 11,930/ $\mathrm{mm}^{3}$; C-reactive protein, $61.8 \mathrm{mg} / \mathrm{dL}$; Ddimer, $1,180 \mathrm{ng} / \mathrm{dL}$; and albumin, $2.4 \mathrm{~g} / \mathrm{dL}$. A chest $\mathrm{CT}$ scan showed a multifocal ground glass pattern associated with thickening of interlobular septa and areas of consolidation, bilateral multifocal distribution, and peripheral, posterior predominance (Fig. 3b).

The patient was treated with oseltamivir, amoxicillin/clavulanic acid, prophylactic enoxaparin, and supportive measures for COVID-19. As gastrointestinal symptoms were controlled, prednisone tapering was accelerated to $20 \mathrm{mg} /$ day, but isoniazid persisted during the entire treatment. The patient's overall status improved progressively, allowing discharge after 7 days.

\section{Discussion}

In December 2019, an outbreak of a novel coronavirus disease (COVID-19) began in Wuhan, China, and it has spread rapidly worldwide [1]. Since then, great concern has been raised regarding the impact of this disease on specific populations at a higher risk for infection, such as IBD patients. Several previous reports have well described the association among immunosuppressive treatment, disease activity, and malnutrition, with a higher frequency and severity of infections in IBD patients $[3,4]$. However, it is still uncertain whether these features constitute additional risks for SARS-CoV-2 infection beyond those 
Fig. 2. Rectosigmoidoscopy revealing areas of spontaneous bleeding and multiple erosions and superficial ulcers, covered with fibrin and hematin.
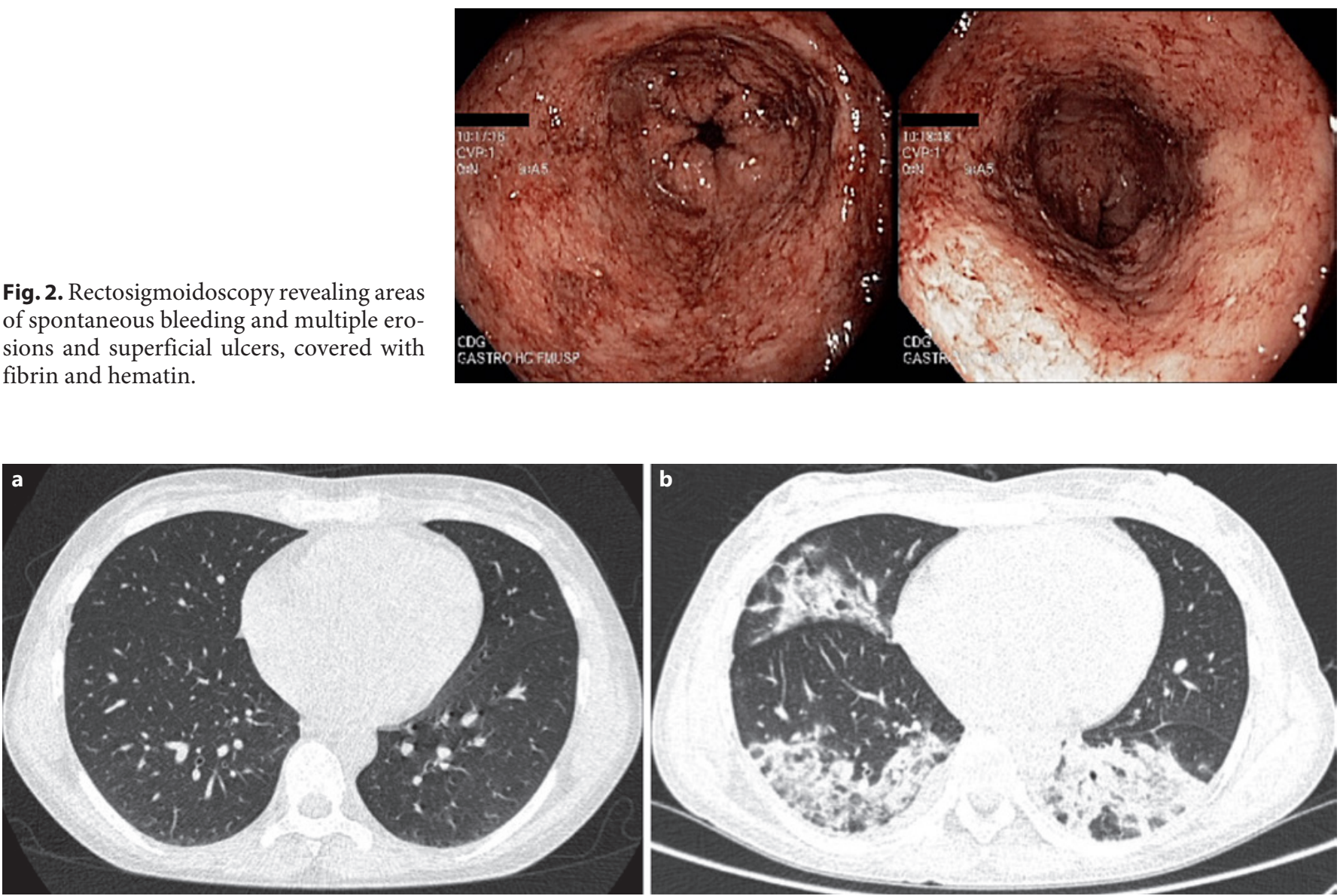

Fig. 3. a Normal chest CT 10 days before COVID-19 diagnosis. b Chest CT after COVID-19 diagnosis showing a multifocal ground glass pattern.

already described for the general population, such as elderly, pregnant, hypertensive, and diabetic patients $[7,8]$. Although data on the clinical characteristics and outcomes of COVID-19 in IBD patients are still very scarce, recent reports suggest that SARS-CoV-2 infection does not occur more frequently in this subgroup of patients than in the general population $[5,8]$.

Recently, an international online database named SECURE-IBD was created in order to monitor and report outcomes of SARS-CoV-2 infection on IBD patients. As of April 8, 2020, thirteen deaths were reported among a total of 382 infected IBD patients [9].

The patient reported herein had a long history of IBD, poorly controlled, and was planning to start biological therapy. Unprecedentedly, after a recent hospitalization for acute severe colitis that responded to steroid treatment, she returned to the hospital with respiratory symptoms secondary to SARS-CoV-2 infection. Considering the incubation

Recovery from COVID-19 Pneumonia in a Patient with Severe Colitis period of up to 14 days [10], we supposed that the infection was possibly acquired in the previous hospitalization.

Despite the presence of high-risk factors for complications of COVID-19 [11], such as severe acute decompensation of IBD and concurrent use of corticosteroids, the patient recovered from COVID-19 pneumonia after 7 days of hospitalization. She was treated with oseltamivir and antibiotics because it was not possible to exclude coinfection by influenza or any bacteria. It remains unclear whether prophylactic enoxaparin played a role in improvement of the patient's prognosis, since some studies have suggested that anticoagulants could prevent coagulopathy in severe COVID-19 [12, 13]. Mazza et al. [14] recently reported the fatal case of an elderly patient who developed SARS-CoV-2 infection during hospitalization for acute severe colitis. Similarly to our case, the patient was on steroids, but probably age was the main factor for the differences in clinical outcomes between these 2 cases, 
since the published data suggest that, in general, young patients have a better prognosis [7].

The international Organization for the Study of Inflammatory Bowel Disease (IOIBD) has issued guidelines stating that IBD patients should continue undergoing their treatment during the COVID-19 outbreak [5]. Although these recommendations are based on expert opinion, it is preferable for IBD patients in clinical remission not to increase their risk of a flare as a consequence of stopping their treatment. Recently, Norsa et al. [15] reported the experience of a tertiary IBD center during the pandemic in a high-prevalence area in Italy. All 522 followed IBD patients were advised to maintain their current treatment and no cases of COVID-19 were reported [15].

In contrast, initiation of immunosuppressive drugs, especially tofacitinib and azathioprine, is of greater concern during the SARS-CoV-2 outbreak and should be avoided as it could increase the risk of viral infections. However, patients in a flare should be treated with the same therapies that would be chosen outside of the pandemic context $[5,8,11]$.

Recently published WHO guidelines suggests that corticosteroids should be avoided in COVID-19 [16]. Evidence from studies in SARS shows that corticosteroid administration might be harmful for patients and has been associated with delayed viral clearance. Their use can be considered in specific patients, such as asthmatics and patients in septic shock [17]. Accordingly, our group decided to taper prednisone rapidly, considering that gastrointestinal symptoms were controlled at that time.

In addition to national recommendations to prevent infection and dissemination of the virus, specific strategies have been implemented worldwide to minimize the potential risk of SARS-CoV-2 infection in the IBD population, such as rescheduling of outpatient visits and elective procedures, tele-health visits to advise patients, and rearrangements in infusion centers $[18,19]$.
This clinical case shows that managing IBD patients and ensuring quality of care during this pandemic can be challenging. We report the first case of COVID-19 pneumonia successfully treated in a patient with acute severe colitis. Emerging data and new reported cases will improve the understanding of the impact of COVID-19 on IBD patients and help to tailor the best strategy to manage patients with a flare during the outbreak.

\section{Statement of Ethics}

The patient gave her written informed consent for publication of data and images. This study was conducted ethically in accordance with the World Medical Association Declaration of Helsinki.

\section{Disclosure Statement}

Dr. Azevedo reports receiving consulting fees from Janssen and lecture fees and travel grant support from Janssen, Takeda, and Abbvie. Dr. Queiroz reports receiving consulting fees from Janssen, lecture fees from Janssen, Takeda, UCB, and Abbvie, and travel grant support from Takeda, Janssen and Abbvie. Dr. Carlos reports receiving lecture fees from Janssen and Boehringer and a travel grant from Janssen, Abbvie, and Takeda. Dr. Damião reports receiving lecture fees from Janssen, Takeda, and Abbvie and a travel grant support from Takeda.

\section{Funding Sources}

The authors declare that no specific funding for this research was received from any agency.

\section{Author Contributions}

All of the authors contributed to the acquisition and analysis of data. Dr. Garcia was a major contributor to the writing of this paper. The authors read and approved the final version of this work.

\section{References}

1 World Health Organization [Internet]. Geneva: World Health Organization; c2020. Available from: https://www.who.int.

2 Zhou F, Yu T, Du R, Fan G, Liu Y, Liu Z, et al. Clinical course and risk factors for mortality of adult inpatients with COVID-19 in Wuhan, China: a retrospective cohort study. Lancet. 2020 Mar;395(10229):105462.

3 Wisniewski A, Kirchgesner J, Seksik P, Landman C, Bourrier A, Nion-Larmurier I, et al. Increased incidence of systemic serious viral infections in patients with inflammatory bowel disease associates with active disease and use of thiopurines. United European Gastroenterol J. 2019 Nov; 8(3):303-13.

4 Zabana Y, Rodríguez L, Lobatón T, Gordillo J, Montserrat A, Mena R, et al. Relevant Infections in Inflammatory Bowel Disease, and Their Relationship With Immunosuppressive Therapy and Their Effects on Disease Mortality. J Crohn's Colitis. 2019 Jul;13(7):828-37.

5 Rubin DT, Abreu MT, Rai V, Siegel CA; International Organization for the Study of In- flammatory Bowel Disease. Management of patients with Crohn's disease and ulcerative colitis during the COVID-19 pandemic: results of an international meeting, Gastroenterology. 2020. doi: https://doi.org/10.1053/j. gastro.2020.04.002.

6 Guan WJ, Ni ZY, Hu Y, Liang WH, Ou CQ, He JX, et al.; China Medical Treatment Expert Group for Covid-19. Clinical characteristics of coronavirus disease 2019 in China. N Engl J Med. 2020 Apr 30;382(18):170820. 
7 Ministério da Saúde. Manual de recomendações para o controle da tuberculose no Brasil. Brasília: Ministério da Saúde, 2019. 364 p.

8 Mao R, Liang J, Shen J, Ghosh S, Zhu LR, Yang $\mathrm{H}$, et al.; Chinese Society of IBD, Chinese Elite IBD Union; Chinese IBD Quality Care Evaluation Center Committee. Implications of COVID-19 for patients with pre-existing digestive diseases. Lancet Gastroenterol Hepatol. 2020 May;5(5):426-8.

9 SECURE-IBD [Internet]. Coronavirus and IBD reporting database [cited 2020 Apr 8]. Available from: https://covidibd.org/.

10 Guo YR, Cao QD, Hong ZS, Tan YY, Chen $\mathrm{SD}$, Jin $\mathrm{HJ}$, et al. The origin, transmission and clinical therapies on coronavirus disease 2019 (COVID-19) outbreak - an update on the status. Mil Med Res. 2020 Mar;7(1): 11.
11 British Society of Gastroenterology [Internet]. British Society of Gastroenterology (BSG) advice for management of inflammatory bowel diseases during the COVID-19 pandemic. 2020 [cited 2020 Mar 22]. Available from: https://www.bsg.org.uk/covid19-advice/bsg-advice-for-management-ofinflammatory-bowel-diseases-during-thecovid-19-pandemic/.

12 Tang N, Bai H, Chen X, Gong J, Li D, Sun Z. Anticoagulant treatment is associated with decreased mortality in severe coronavirus disease 2019 patients with coagulopathy. J Thromb Haemost. 2020 Mar; 18(5):1094-99.

13 Tang N, Li D, Wang X, Sun Z. Abnormal coagulation parameters are associated with poor prognosis in patients with novel coronavirus pneumonia. J Thromb Haemost. 2020 Apr; 18(4):844-7.

14 Mazza S, Sorce A, Peyvandi F, Vecchi M, Caprioli F. A fatal case of COVID-19 pneumonia occurring in a patient with severe acute ulcerative colitis. Gut. 2020. doi: 10.1136/ gutjnl-2020-321183
15 Norsa L, Indriolo A, Sansotta N, Cosimo P, Greco S, D'Antiga L. Uneventful course in IBD patients during SARS-CoV-2 outbreak in northern Italy. Gastroenterology. 2020. doi: 10.1053/j.gastro.2020.03.062.

16 World Health Organization. Clinical management of severe acute respiratory infection (SARI) when COVID-19 disease is suspected: interim guidance, 13 March 2020. Geneva: World Health Organization; 2020.

17 Stockman LJ, Bellamy R, Garner P. SARS: systematic review of treatment effects. PLoS Med. 2006 Sep;3(9):e343.

18 Fiorino G, Allocca M, Furfaro F, Gilardi D, Zilli A, Radice S, et al. Inflammatory bowel disease care in the COVID-19 pandemic era: the Humanitas, Milan experience. J Crohn's Colitis. 2020. doi: 10.1093/ecco-jcc/jjaa058.

19 Danese S, Cecconi M, Spinelli A. Management of IBD during the COVID-19 outbreak: resetting clinical priorities. Nat Rev Gastroenterol Hepatol. 2020. doi: 10.1038/s41575-0200294-8. 